\title{
Analysis of ABC1 protein family members in Lepidium apetalum seeds and the expression of $L a A b c 1$ in seedlings in response to abiotic stresses
}

\author{
Q.L. YANG ${ }^{1}$, Z.Y. CHEN ${ }^{1,2}$, H. LU ${ }^{3}$, H.T. XIE ${ }^{1}$, J.Y. LI ${ }^{1}$, Y. DU ${ }^{1}$, S.C. HAN ${ }^{2}$, H.P. ZHAO ${ }^{1,2}$, \\ and H.X. ZHAO ${ }^{1 *}$ \\ College of Life Science, Xinjiang Normal University, Xinjiang Urumqi 830054, P.R. China ${ }^{1}$ \\ College of Life Sciences, Beijing Normal University, Beijing 100875, P.R. China ${ }^{2}$ \\ Dough academy, Hotan Teachers College, Xinjiang Hotan 848000, P.R. China ${ }^{3}$
}

\begin{abstract}
To study the biological function of activity of bcl complex (ABC1) proteins in Lepidium apetalum Willd., genes encoding $\mathrm{ABC} 1$ family proteins were identified from the seed transcriptome. The sequence most closely related to germination at a low temperature was selected and gene expressions in response to low temperature stress further studied. The results show that $21 A B C 1$ genes were expressed in seeds germinating at the low temperature: 4 genes were upregulated, 6 were downregulated, and 11 were not significantly different from controls. The results of fluorescence quantification of the lowtemperature stress on the seedlings of 7-d-old L. apetalum showed that seven genes were up-regulated, six genes were down-regulated, and eight genes had no significant difference. Real-time quantitative PCR results show that under the low temperature stress, the expression of the $L a A b c 1-3$ gene increased, but its expression decreased after some time. The expression of this gene increased again after removing the low temperature stress. The expression of $L a A b c 1-21$ gene in L. apetalum seedlings showed a trend of decreasing first and then increasing. The LaAbcl-3 gene was insensitive to salt stress. Expression of the LaAbcl-21 gene was significantly up-regulated during the salt stress. Under osmotic stress, the expression of the LaAbcl-3 gene was down-regulated, and the expression was negatively correlated with polyethylene glycol (PEG-6000) concentration. Under the PEG-6000 treatment, the expression of the LaAbc1-21 gene was significantly up-regulated, and the expression was positively correlated with concentration. These results provide a basis for further analysis of the role of the $A B C 1$ genes in the stress resistance of L. apetalum.
\end{abstract}

Additional key words: LaAbcl-3 and LaAbcl-21 genes, low temperature, osmotic stress, salt stress.

\section{Introduction}

Lepidium apetalum Willd. is a cruciferous oleaginous plant, widely distributed, and with medicinal values. The ability to adapt to low temperatures in early spring is strong, and it can germinate when ice and snow begin to melt (Li et al. 2016). It is a pioneer plant in the process of ecosystem succession. This growth feature has gradually become the focus of research in many scientific research projects (Meng et al. 2008). The seeds are dormant at low temperature, but they can tolerate the low temperature for germination and seedling growth after high temperature treatment to break dormancy (Zhao et al. 2010). The ecotype in northern Xinjiang has been reported to grow at $0-5{ }^{\circ} \mathrm{C}$ in some cases (Mao and Zhang 1994). In a study of seed germination characteristics, it was found that the low-temperature germination of $L$. apetalum proceeds as follows, at temperatures of $0-5^{\circ} \mathrm{C}$, the initial germination of seeds can occur, but there is a stagnation period. Interestingly, brief exposure to a higher temperature (e.g., $25^{\circ} \mathrm{C}$ for $50 \mathrm{~min}$ ) allows the seeds to circumvent the stagnation period (Yang et al. 2015). When germinated seeds are again exposed to low temperatures, they can tolerate low temperatures and continue to germinate and grow (Zhao et al. 2010). Considering the response of seeds to temperature, the transcriptome of seeds under stagnant low temperature was compared with that of seeds after short-term high temperature treatment, and the differentially expressed genes were identified (Young et al. 2018, Smita et al. 2020). In addition to low temperature, plants also suffer from drought and salt stress at the stage of seed germination and seedling growth. They

Submitted 4 June 2020, last revision 8 July 2020, accepted 13 July 2020.

Abbreviations: ABC1 - activity of bcl complex; GO - gene ontology; KOG - eukaryotic ortholog groups; Nr - non-redundant; PEG polyethylene glycol.

Acknowledgments: This work was supported by grants from the Open Project Fund of Xinjiang Uygur Autonomous Region Key Laboratory, the National Natural Science Foundation of China (31660079 and 31460041), and Xinjiang Normal University Postgraduate Research Innovation Fund Project.

* Corresponding author; e-mail: 954843437@qq.com 
can be used as ideal materials to study the mechanism of seedling tolerance to low temperature. Considering the harsh growth environment of $L$. apetalum, activity of bcl complex $(\mathrm{ABC} 1)$ proteins have recently been investigated. $\mathrm{ABC} 1$ family proteins play an important role in abiotic stress tolerance in plants, including cold, osmotic, and salt stresses (Wang et al. 2004, Gao et al. 2011a,b, 2012, Yuan et al. 2014).

Activity of bcl complex (ABC1) is a member of the protein kinase superfamily and is involved in physiological regulation in prokaryotes and eukaryotes (Trumpower, 1981, Trumpower, 1990, Ernster and Forsmark 1993, Leonard et al. 1998, Villalba and Navas 2000, Michal et al. 2008). The prototype of this family was isolated from Saccharomyces cerevisiae and was named due to its role in the suppression of a defect in cytochrome $b$ mRNA translation and maintainance of the activity of bcl complex in the mitochondrial respiratory chain (Bousquet et al. 1991). Evolutionary analysis shows that the $\mathrm{ABCl}$ protein located in the mitochondria and the chloroplast $\mathrm{ABCl}$ egg F1 have different origins (Leonard et al. 1998). Studies in yeast, E. coli, and other species including humans have shown that the mitochondrial and prokaryotic $\mathrm{ABCl}$ proteins regulate the biosynthesis of ubiquinone (David et al. 1998, Do et al. 2001, Hsieh et al. 2004, Mollet et al. 2008, Tauche et al. 2013) and participate in respiratory electron transport and anti-oxidative stress (Trumpower 1981, Ernster and Forsmark 1993, Villalba and Navas 2000). Michal et al. (2008) found that the Arabidopsis chloroplast $\mathrm{ABCl}$ protein AtOSAl is a cadmium and oxidative stress response factor, and the plants are subjected to oxidative stress after mutation of its coding gene.

In Arabidopsis, ABC1 gene family members ATOSA1, AtSIA1, and AtACDOI can be widely involved in response to abiotic stresses such as chromium, oxidation, and high salt (Michal et al. 2008, Yang et al. 2012a,b,c). Wheat $T a A B C 1 L$ gene has also been proved to be a response gene to various abiotic stresses such as high salt, high osmolality, low temperature and ABA (Wang et al. 2004, 2011). Of the $14 A B C 1$ gene family members in rice, 7 were induced or suppressed by low temperature stress, 8 responded to salt stress, and 4 were suppressed by drought treatment (Gao et al. 2011b, Yang et al. 2012a).

In the previous study, we found that seedlings of L. apetalum can tolerate low temperature and high salinity. Moreover, the expressions of some functional genes, such as LaLEAs (Yang et al. 2020), show a significant correlation with stress. However, it is not clear whether $\mathrm{ABC} 1$ family transcription factors also respond to abiotic stresses. Therefore, we will screen and obtain the coding sequence of $\mathrm{ABC} 1$ family and analyze the relationship between the expression and stress.

\section{Materials and methods}

Plant, treatments, and related data sources: Lepidium apetalum Willd. ripe seeds were collected from Liyushan mountain, Urumqi, Xinjiang, northern China. After drying at room temperature, the seeds were stored at $4{ }^{\circ} \mathrm{C}$. Seeds were treated with $98 \%(\mathrm{~m} / \mathrm{v})$ sulfuric acid to remove the seed coat, rinsed with distilled water several times, placed on wet filter paper, and left at a temperature of $24-26^{\circ} \mathrm{C}$, a 16-h $\cdot$ photoperiod, an irradiance of 400 to $650 \mu \mathrm{mol} \mathrm{m}{ }^{-2} \mathrm{~s}^{-1}$, and a relative humidity of $60 \pm 5 \%$ to germinate for 1 week. The 7-d-old seedlings were obtained for cloning the cDNA of the $L a A b c 1$ gene, and to verify the response of the $L a A b c 1$ gene to abiotic stresses as was perviously described (Yang et al. 2020). The ABC1 sequence information on L. apetalum seeds was obtained from the transcriptome sequencing results (Zhou et al. 2016). Gene sequence information (taxonomy: Lepidium apetalum.) for other species was obtained from the NCBI database (https:// www. ncbi.nlm.nih.gov).

Screening for $\mathbf{A B C 1}$ protein family genes: $A B C 1$ gene related sequences were selected from the transcriptome data of $L$. apetalum seeds with $\mathrm{ABC} 1$ as the key word. Each sequence screened was analyzed in the NCBI database for $B L A S T$ analysis. Only those sequences that are highly homologous (consistency greater than $85 \%$ ) with the sequences annotated as $\mathrm{ABC} 1$ in $N C B I$ and contain the conservative domain of $\mathrm{ABC} 1$ can be identified as members of the $\mathrm{ABC} 1$-related family.

Functional determination of $\mathrm{ABC} 1$ protein family genes was carried out using the gene ontology ( $G O$; http:// geneontology.org/) and eukaryotic ortholog groups (KOG; https://blast.ncbi.nlm.nih.gov/Blast.cgi) databases, as well as $N C B I$ non-redundant ( $N r$; ftp://ftp.ncbi.nlm.nih.gov/ blast/db) protein analyses.

Extraction of total RNA from seedlings of Lepidium apetalum: A TRIzol reagent was used to extract total RNA from 7-d-old seedlings, treatment with DNase I to remove DNA contamination in samples. The integrity and quality of the total RNA (1.2\% agarose gel electrophoresis), the purity of the RNA (UV spectrophotometer) were tested, and, finally, the Agilent 2100 (Applied Biosystems, Beijing, China) was used to accurately detect the integrity of the RNA. The cDNA synthesis was carried out using a reverse transcription kit (a RevertAid First Strand cDNA synthesis kit) according to the manufacturer's instructions. The cDNAs were stored at $-80{ }^{\circ} \mathrm{C}$ until further use.

Differential expression analysis of $\mathrm{ABC} 1$ protein family genes before and after low-temperature germination: Based on the fragments per kilobase of transcript per million fragments mapped method, the differential expression of $\mathrm{ABC} 1$ protein coding gene in transcriptome was analyzed. Up or down regulation of genes more than 0.5 - 1.5 times is considered to be differentially expressed. Target genes of $L$. apetalum seeds with significant differences in expression before versus after germination at low temperature were identified.

Cloning and sequence analysis of the full- length

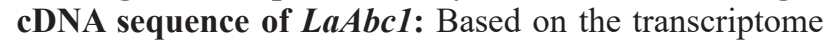
sequencing results of the seeds, specific primers for the amplification of $L a A b c 1$ were designed (Table 1 Suppl.). Using the cDNA of $L$. apetalum seedlings as a template, 
the full-length sequence of the $L a A b c 1$ gene was amplified (commissioned by the Beijing PMAD company for sequencing). ClustalX (Thompson et al. 1999) software was used to compare these sequences with the transcriptome sequences. The NCBI database was used to analyze sequence homology. By using MEGA 5 software (Tamura et al. 2011) to align amino acid sequences, and based on maximum likelihood (ML) method, the phylogenetic tree was constructed through 1000 bootstrap tests. The properties of the protein were analyzed by ProtParam and the secondary structure was predicted using ExPASy (http://web.expasy.org/protparam/). The Swiss-model (https://swissmodel.expasy.org/) was used to predict the tertiary structure of the proteins.

Expression of $L a A b c 1$ in response to abiotic stresses: Analysis of the expression of $21 \mathrm{LaAbcl}$ genes in L. apetalum seedlings was performed by fluorescence quantitative method (for detail see Yang et al. 2020). For low temperature stress, 7-d-old seedlings were placed at $0^{\circ} \mathrm{C}$ for $0,1,6,12,24,36$, and 48 h. For salt stress, using distilled water as the medium, seedlings were cultured in different $\mathrm{NaCl}$ concentrations $(0,150$, and 300 $\mathrm{mM}$ ) for $7 \mathrm{~d}$. For osmotic stress, using distilled water as the medium, 7-d-old seedlings were cultured in medium containing 0,20 , and $30 \%(\mathrm{~m} / \mathrm{v})$ polyethylene glycol (PEG $6000)$. Seedling recovery at $25^{\circ} \mathrm{C}, 0 \mathrm{mM} \mathrm{NaCl}$, and $0 \%$ PEG 6000 was performed for 12, 24, and $48 \mathrm{~h}$ after 48 -h low temperature, salt, and osmotic stresses. The expression of the $L a A b c 1$ gene in response to cold, salt, and osmotic stress was analyzed by fluorescence quantitative method. The seedlings grown in media without any additions and at room temperature were used as control plants.

Statistical analysis: The data were analyzed by one-way ANOVA using SPSS 20 (IBM, Armonk, NY, USA). The significant differences among treatments were tested based on the least significant difference (LSD) at $P \leq 0.05$.

\section{Results}

Based on the transcriptome sequencing results of L. apetalum seeds, 29 sequences containing the keyword ABC1 were identified. Following removal of redundant sequences, $21 A B C 1$ gene sequences were identified in $N C B I N r$ database for L. apetalum. A phylogenetic tree was constructed using $21 \mathrm{ABC} 1$ protein enconding genes sequences, which show that all of them belong to the $\mathrm{ABC} 1$ protein family (Fig. 1). The longest and shortest sequences were 2484 and $264 \mathrm{bp}$, respectively. Of the $21 \mathrm{ABC1}$ sequences, five encoded complete open reading frames.

The predicted functions of the products encoded by the $21 A B C 1$ genes of $L$. apetalum were obtained by $G O$ analysis. In total, $15 A B C 1$ genes were annotated to the biological process category, 14 to the molecular function category, and 5 to the cellular component category (Fig. $2 A$ ). Among the $21 A B C 1$ genes, only 5 were annotated to the three ontologies. It is speculated that these five genes

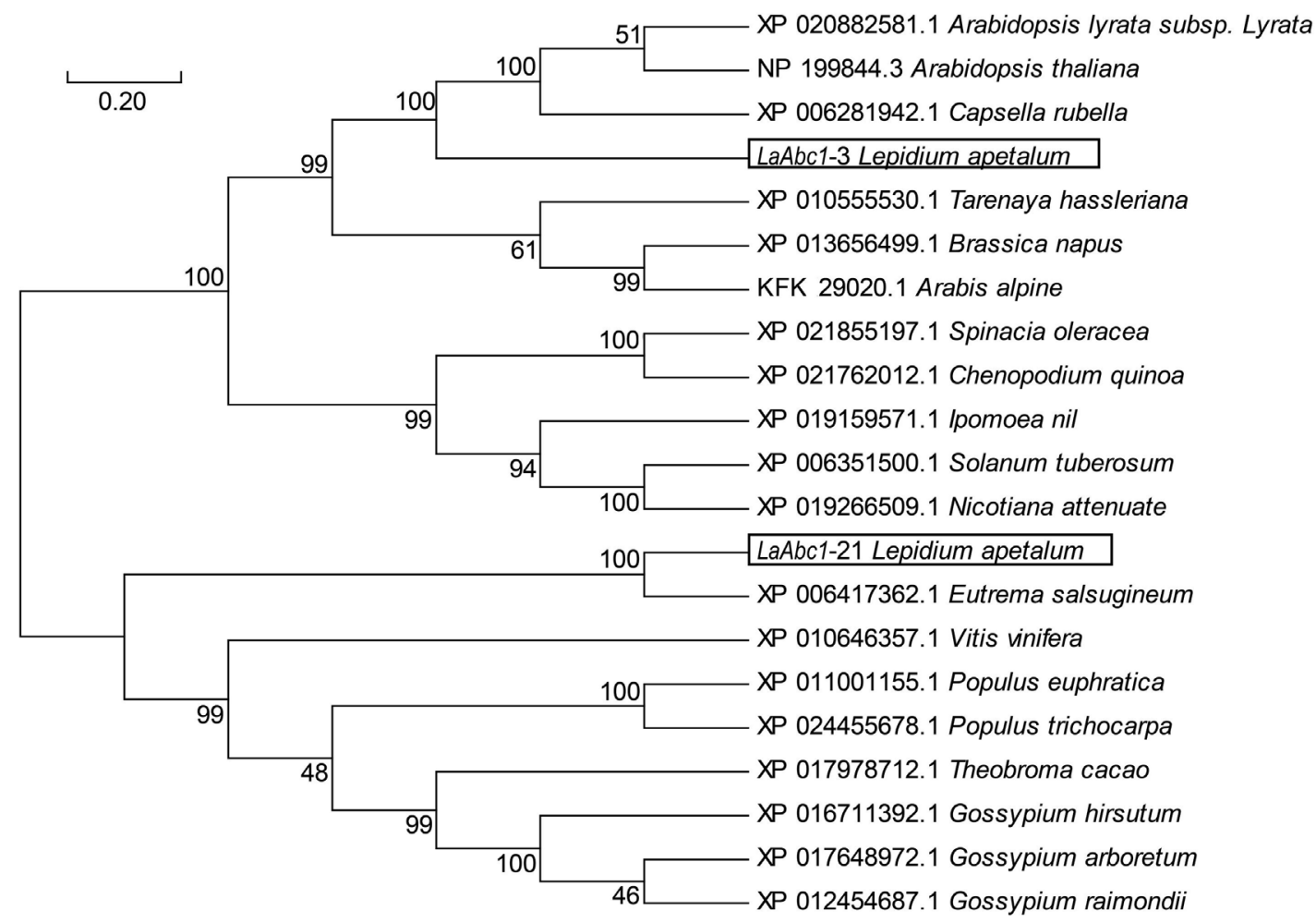

Fig. 1. A phylogenetic tree of Lepidium apetalum activity of bcl complex $(\mathrm{ABC} 1)$ with the amino acid sequences of $\mathrm{ABC} 1$ from other species. The numbers at nodes represent bootstrap values based on 1000 replicates. The scale represents genetic distance. NP - nonprotein, XP - predicted model protein, KFK - hypothetical protein. 


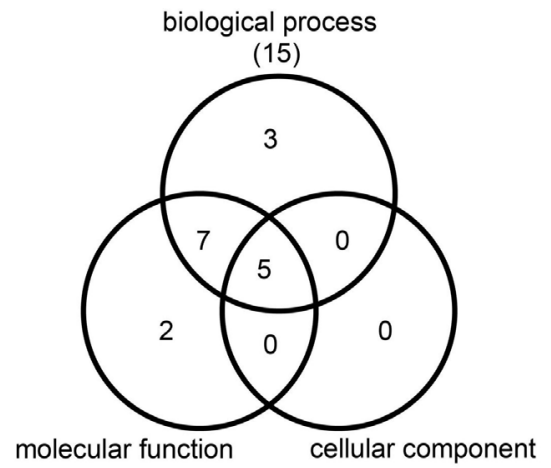

(14)

(5)

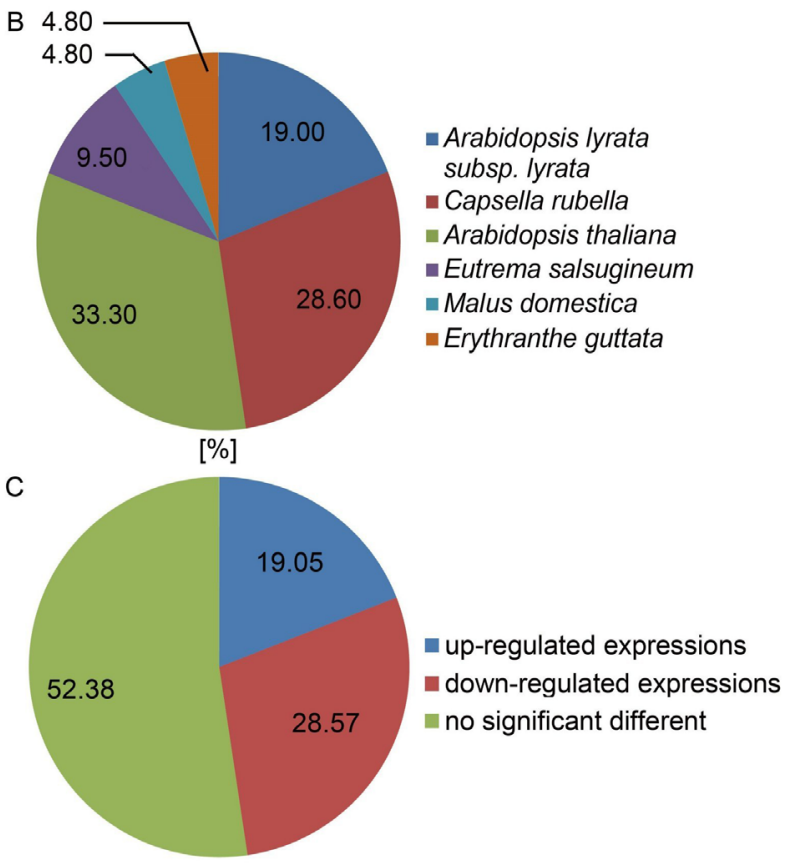

[\%]

Fig. 2. Lepidium apetalum activity of bcl complex (ABC1) protein family unigene distribution based on the gene ontology analysis $(A)$ and $N C B I N r$ database $(B)$. Relative expression difference between controls and seedlings stressed by low temperature $\left(4^{\circ} \mathrm{C}\right.$ for $\left.9 \mathrm{~d}\right)(\mathrm{C})$.

may have multiple functions in biological development and molecular stress resistance. $80 \%$ of the $21 \mathrm{ABC} 1$ transcriptional factors were annotated to biological processes and molecular functions, indicating that these sequences are involved in the growth and development of L. apetalum and play an important molecular function in adaptation to stress.

Analysis of the NCBI Nr database indicates that the identified $\mathrm{ABC} 1$ protein family genes were homologous to varying degrees with the known genes of 6 related species (Fig. 2B), 33.3\% were homologous with the Arabidopsis thaliana; $28.6 \%$ with the Capsella rubella, and $19 \%$ with Arabidopsis lyrata subsp. lyrata.

Based on the conserved sites, the plant $\mathrm{ABC} 1$ protein sequences were grouped into 26 functional components using the $K O G$ database. In this study, fourteen $\mathrm{ABC} 1$ protein family gene sequences isolated from the seeds of
L. apetalum were functionally annotated in $K O G$. Only one sequence was reported, which was annotated as O (posttranslational modification, protein turnover, chaperones); the other seven sequences were not annotated in the $K O G$ database (Table 2 Suppl.).

The transcriptional data of $\mathrm{ABC} 1$ protein family genes of $L$. apetalum seeds were analyzed before and after germination stagnation at low temperature (Fig. 3). We conducted real-time fluorescence quantitative PCR to validate the transcriptome data and to analyze the gene expression changes of randomly selected genes. Although the expressions of selected genes were different between transcriptomics and quantitative PCR, the trends of expression were the same (Fig. 1 Suppl.). Four upregulated and six downregulated genes were identified, the remaining eleven genes showed no significant difference based on transcriptional data (Fig. 2C). The c13379 g1 and c21578_g1 sequences were selected for further analysis based on the relationship between their expression and abiotic stresses. The sequences were named LaAbc1-3 and LaAbc1-21 in this study.

Fluorescence quantitative analysis shows that LaAbc1-2, LaAbc1-3, LaAbc1-5, LaAbc1-6, LaAbc1-8, $L a A b c 1-18$, and $L a A b c 1-21$ were up-regulated in 7-d-old L. apetalum seedlings treated at $0^{\circ} \mathrm{C}$ for $0,1,2,4,8,12,24$, and $48 \mathrm{~h}$. After low temperature treatment, the expression of these genes increased significantly compared with the control group $\left(0^{\circ} \mathrm{C}\right.$ treatment for $\left.0 \mathrm{~h}\right)$. The expression of the LaAbc1-3 and LaAbc1-5 genes increased first and then decreased. The LaAbcl-5 increased within $12 \mathrm{~h}$ when it reached its maximum, which was 14-times higher than in the control group. This result indicated that the increase of $L a A b c 1-5$ expression might be related to the cold resistance of $L$. apetalum seedlings. After $12 \mathrm{~h}$, the expression of the LaAbcl-5 gene showed a downward trend, but its expression was still higher than that of the control group. The rapid response of the $L a A b c 1-6$ gene to low temperature stress made the seedlings adapt quickly to the adverse environment. With the prolongation of treatment time, the expression of the LaAbcl-6 gene was significantly up-regulated, indicating that the gene was closely related to low temperature stress tolerance of the L. apetalum seedlings. The expression of the LaAbcl-2, $L a A b c 1-8$, and $L a A b c 1-21$ genes increased rapidly after $1-2 \mathrm{~h}$ of low temperature treatment to enhance the adaptability of plants to low temperature, and then downregulated expression of these genes may indicate that these genes have accumulated a large number of synthetic protein templates in a short time. The expression of the LaAbc1-21 gene decreased first and then increased, the decrease of expression may be due to the damage of L. apetalum seedlings by rapid low temperature treatment. After a short time of inhibition, the LaAbcl-21 gene expression increased (Fig. 2 Suppl.).

The results show that the expression of LaAbc1-7, LaAbc1-9, LaAbc1-12, LaAbc1-13, LaAbc1-15, LaAbc1-16, LaAbc1-19, and LaAbcl-20 in ABC1 transcription factors had no significant difference after low temperature treatment. Compared with the control group, the expression of genes decreased slightly with 


\begin{tabular}{|c|c|c|c|c|c|}
\hline GENE & $\begin{array}{l}\text { SERIAL } \\
\text { NUMBER }\end{array}$ & LENGT & HOMOLOGOUS PROTEINENCODING GENE & $\begin{array}{l}\text { CONTROL } \\
\text { MEAN }\end{array}$ & $\begin{array}{l}\text { EXPERIMENTAL } \\
\text { MEAN }\end{array}$ \\
\hline$A b c 1-1$ & c6383_g1 & 1047 & Arabidopsis lyrata subsp. lyrata serine/threonine-protein kinaseabkC & & \\
\hline$A b c 1-2$ & c15065_g1 & 597 & Arabidopsis lyrata subsp. lyrata UPF0051 protein ABCl8 & & \\
\hline$A b c 1-3$ & c13379_g1 & 1470 & Arabidopsis lyrata subsp. Iyrata aafF domain-containing protein kinase 1 & & \\
\hline$A b c 1-4$ & c9338_g1 & 714 & Camelina sativea aarF domain-containing protein kinase2 & & \\
\hline$A b c 1-5$ & c22062_g1 & 2442 & Arabidopsis lyrata subsp. lyrata uncharacterized protein sll0005 & & \\
\hline$A b c 1-6$ & c21890_g1 & 2061 & Arabidopsis thaliana protein kinase superfanmily protein (ACDO1) & & \\
\hline$A b c 1-7$ & c12914_g1 & 534 & Eutrema salsugineum aarF domain-containing protein kinasa1 & & \\
\hline$A b c 1-8$ & c22903_g2 & 2139 & Capsella rubella ABC1-like kinase & & \\
\hline$A b c 1-9$ & c22279_g1 & 2484 & Arabidopsis thaliana protein kinase superfamily protein ATATH 8 & & \\
\hline$A b c 1-10$ & c22062_g2 & 264 & Camelina sativa uncharacterized protein sll0005-like & & \\
\hline Abc1-11 & c8932_g2 & 318 & Capsella rubella uncharacterized protein slr0889 & & \\
\hline$A b c 1-12$ & c10431_g1 & 588 & Camelina sativa UPF0051 protein ABCl8 & & \\
\hline Abc1-13 & c22596_g1 & 1146 & Camelina sativa aarF domain-containing protein kinase At1g71810 & & \\
\hline Abc1-14 & c22316_g1 & 996 & Arabidopsis lyrata subsp. lyrata uncharacterized protein sir 1919 & & \\
\hline$A b c 1-15$ & c19216_g1 & 1689 & Arabidopsis thaliana ATATH9 & & \\
\hline$A b c 1-16$ & c20945_g2 & 1152 & Arabidopsis thaliana $\mathrm{ABC} 1$ family protein & & \\
\hline$A b c 1-17$ & c23357_g1 & 1740 & Camelina sativa aafF domain-containing protein kinase At5g05200 & & \\
\hline$A b c 1-18$ & c21106_g1 & 312 & Capsella rubella aarF domain-containing protein kinaes1 & & \\
\hline$A b c 1-19$ & c8932_g1 & 327 & Arabidopsis taliana At4g24810 & & \\
\hline$A b c 1-20$ & c18258_g1 & 2127 & Capsella rubella protein $\mathrm{ABC}$ transporter1 & & \\
\hline$A b c 1-21$ & c21578_g1 & 1959 & Eutrema salsugineum aarF domain-containing protein kinase2 & & \\
\hline
\end{tabular}

Fig. 3. Relative expression analysis of activity of bcl complex ( $\mathrm{ABC} 1)$ protein family genes before (control mean) and after (experimental mean) seed germination at low temperature $\left(4{ }^{\circ} \mathrm{C}\right.$ for $\left.9 \mathrm{~d}\right)$ in Lepidium apetalum based on transcriptome analysis.

the prolongation of low temperature treatment time, but the expression was similar, which indicated that the genes were not obvious in response to low temperature stress, and might be constitutive genes (Fig. 2 Suppl.).

The results show that $L a A b c 1-1, L a A b c 1-4, L a A b c 1-10$, LaAbc1-11, LaAbc1-14, LaAbc1-17 of ABC1 transcription factors were down-regulated in the L. apetalum seedlings after low temperature treatment at $0{ }^{\circ} \mathrm{C}$ (Fig. 2 Suppl.). These down-regulated genes can reduce the decomposition of some proteins to reduce the hydrolysis of amino acids and protect plants from ammonia poisoning, thus increasing the tolerance of plants to low temperature stress.

Homologous cloning was used to amplify the bands with the expected gene size. The LaAbcl-3 encodes 478 amino acids (Fig. 3 Suppl.). The LaAbc1-3 ProtParam analysis shows that the gene had a length of $54063.82 \mathrm{~kb}$, the protein a molecular mass of $54.07 \mathrm{kDa}$, a theoretical isoelectric point of 9.08, a molecular formula of $\mathrm{C}_{2420} \mathrm{H}_{3871} \mathrm{~N}_{671} \mathrm{O}_{685} \mathrm{~S}_{23}$, and an instability index of 43.65. It belonged to unstable proteins with an average hydrophilic coefficient of -0.189 , and it was a hydrophilic protein. The LaAbc1-21 encoded 630 amino acids (Fig. 4 Suppl.). The ProtParam analysis shows that a length of the gene was
$71380.62 \mathrm{~kb}$, a protein molecular mass of $71.39 \mathrm{kDa}$, a theoretical isoelectric point of 9.83, a molecular formula of $\mathrm{C}_{3226} \mathrm{H}_{5066} \mathrm{~N}_{912} \mathrm{O}_{878} \mathrm{~S}_{22}$, an instability index of 41.44 , (belonging to unstable proteins), a fat index of 81.94 , and an average coefficient of hydrophilicity of -0.188 ; it was a hydrophilic protein.

The protein domain analysis of LaAbcl-3 using the SMART (http://smart.embl-heidelberg.de/) software shows that the protein encoded by this gene had a highly conserved protein kinase functional domain STYKc family from 132 to 299 (Fig. 5 Suppl.). The LaAbc1-21 protein domain analysis shows that the protein encoded by this gene had a transmembrane domain on peptide segments 171 to 193 , which might be closely related to abiotic stress tolerance in the L. apetalum seedlings (Fig. 5 Suppl.). The protein sequence encoded by $L a A b c 1-3$ had an $\mathrm{ABCl}$ characteristic domain between amino acids 122 and 240 and an AARF conserved domain between amino acids 58 and 430 . The protein sequence encoded by $L a A b c 1-21$ had an $\mathrm{AABCl}$ characteristic domain between amino acids 877 and 1200 and an AAR conserved domain between amino acids 625 and -1854 .

Prediction of secondary structure of protein encoded 


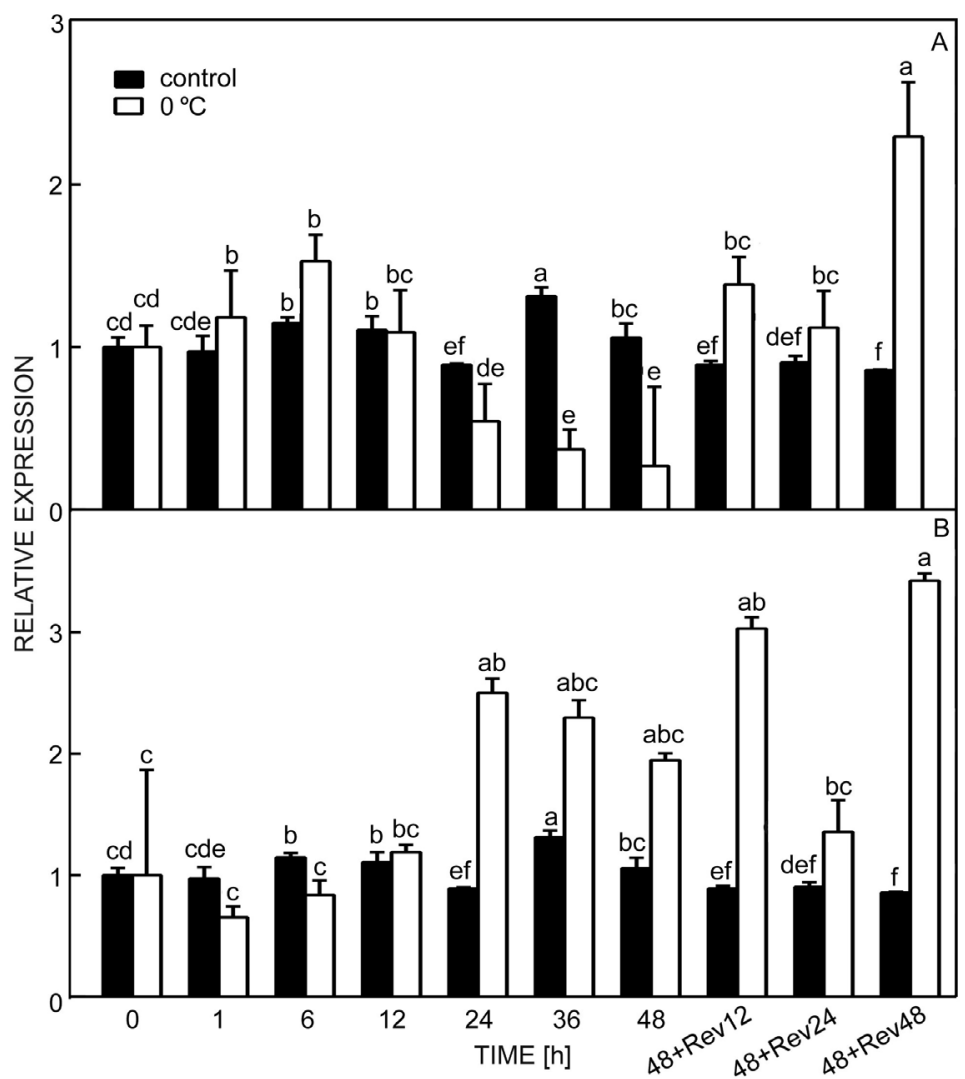

Fig. 4. Lepidium apetalum activity of bcl complex $(A B C 1)$ relative expression in seedlings under a short-term (48 h) low temperature stress $\left(0{ }^{\circ} \mathrm{C}\right)$ and recovery at $25{ }^{\circ} \mathrm{C}$ (Rev). $A-L a A b c 1-3, B-L a A b c 1-21$. Means \pm SEs, $n=3$, different letters indicate significant differences among treatments based on the least significant difference at $P \leq 0.05$.

by the L. apetalum LaAbc1-3 gene showed that $60.25 \%$ of the protein was $\alpha$-helix and $26.36 \%$ was irregular curl. Three-dimensional spatial structure prediction of L. apetalum LaAbcl-3 encoding proteins was carried out in univegetable by Swiss-model online software (Fig. 5 Suppl.). The results showed that the protein was mainly composed of $\alpha$-helix and irregular curl, and the proportion of extended chain and $\beta$-angle was small, which was basically consistent with the secondary structure of the protein.

Prediction of secondary structure of protein encoded by the L. apetalum LaAbc1-21 gene showed that the protein was mainly $\alpha$-helix (54.13\%) and irregular curl $(32.54 \%)$ (Fig. 5 Suppl.). The proportion of elongation chain and $\beta$-corner was small, which was basically consistent with the secondary structure of the protein.

Analysis of homology alignment of the L. apetalum LaAbcl-3 gene coding amino acid sequence with Arabidopsis lyrata subsp. lyrata, Arabidopsis thaliana, and Capsella rubella coding amino acid sequence showed low conservativeness at $\mathrm{N}$ and $\mathrm{C}$ ends of the sequence. The conservative genotype from 290 to 340 amino acids is higher, which may be related to the functional consistency of the gene in different species. The phylogenetic tree of LaAbcl-3 and its related species ABC1 amino acid sequence was constructed by MEGA 5 (Fig. 3). Lepidium apetalum LaAbc1-3 is similar to Arabidopsis lyrata subsp, Arabidopsis thaliana, Capsella rubella, and Brassica napus and other Brassicaceae plants. The results showed that the gene was conservative, but it formed a single branch, indicating that the functions of homologous proteins were different.

The homologous alignment of the $L$. apetalum LaAbc1-21 gene coding amino acid sequence with Eutrema salsugineum, Gossypium, Populus euphratica, and Vitis vinifera coding amino acid sequence was analyzed. The low conservativeness at the near $\mathrm{C}$ end of the sequence may be related to the functional differences of the gene in different species. The N-terminal conservative type is higher, which may be related to the specific function of the gene in different species. The phylogenetic tree of $L a A b c 1-21$ and its related species $\mathrm{ABC} 1$ amino acid sequence was constructed by MEGA 5 (Fig. 3). The homology of $L$. apetalum LaAbc1-21 is only close to that of the genus Eutrema salsugineum, Gossypium hirsutum, Populus euphratica, and cocoa and grapes form another big branch. The signal peptides of LaAbc1-3 and LaAbc1-21 proteins were predicted and analyzed. The two proteins did not have signal peptides, indicating that they were not secretory proteins (Fig. 6 Suppl.).

Quantitative fluorescence analysis of different tissues of wild L. apetalum at the same growth period showed that the LaAbcl-3 gene was up-regulated in the old stem, and the LaAbc1-21 gene had no tissue specificity (Fig. 7 Suppl.).

The expression of $L a A b c 1-3$ gene increased first, then 


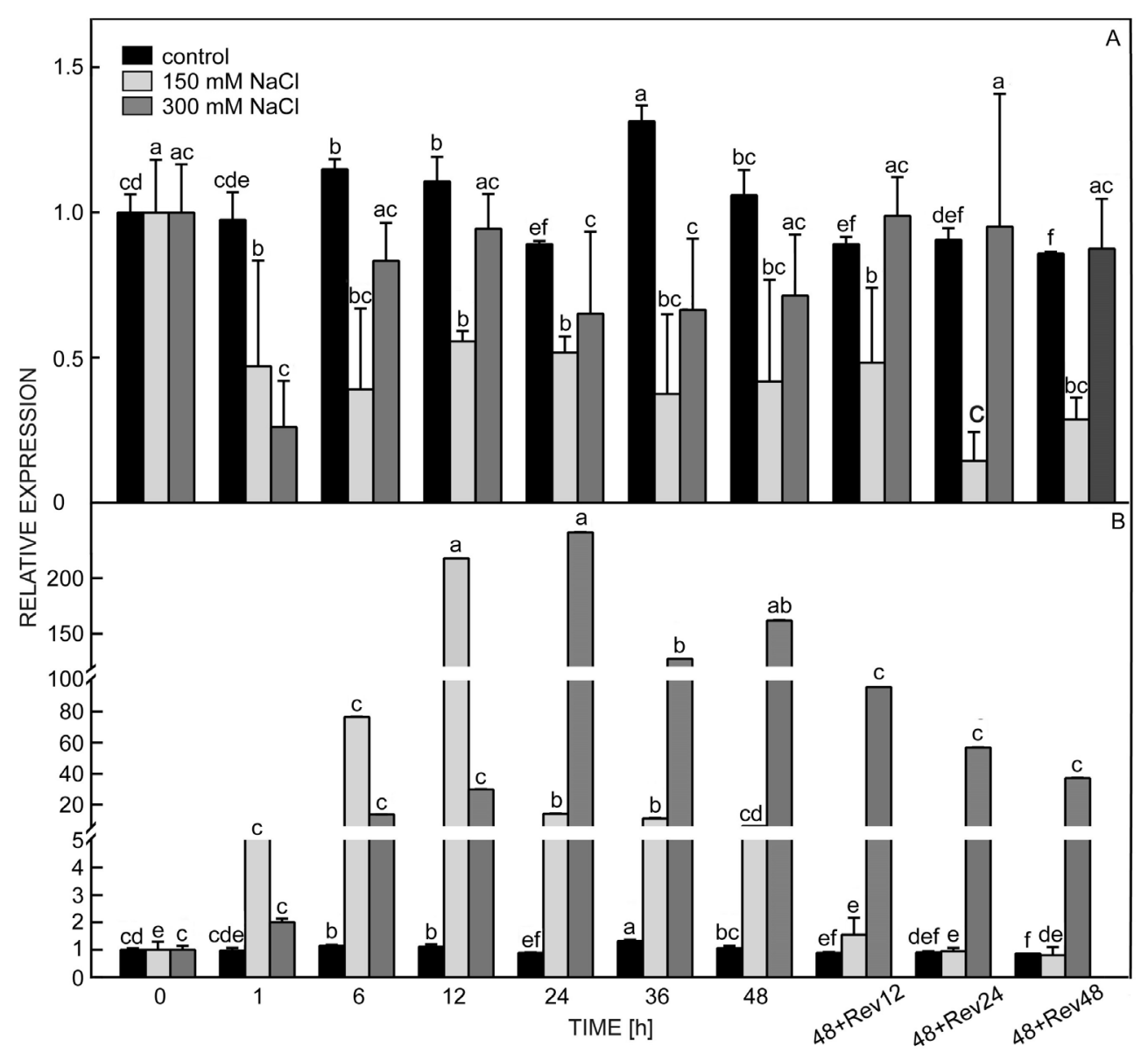

Fig. 5. Lepidium apetalum activity of bcl complex $(A B C 1)$ relative expression in seedlings treated with different concentrations of $\mathrm{NaCl}$ for 0 to $48 \mathrm{~h}$ and during recovery for 12 to $48 \mathrm{~h}$ (Rev). $A$ - LaAbcl -3, $B$ - LaAbcl-21. Means $\pm \mathrm{SEs}, n=3$ samples from 40 - 50 plants; different letters indicate significant differences among treatments based on the least significant difference at $P \leq 0.05$.

decreased, and then increased during low temperature treatment and recovery, and reached the maximum at $6 \mathrm{~h}$ of cold treatment. After $6 \mathrm{~h}$, the expression of the gene decreased, and the expression of the gene during recovery was significantly higher than that in the control group, it indicated that low temperature stress had not been completely eliminated, and it would take some time for the seedlings to return to optimum conditions. The expression of the $L a A b c 1-21$ gene decreased at 0 - $1 \mathrm{~h}$ after cold treatment, and increased after $1 \mathrm{~h}$, after removing the stress. The expression was still up-regulated compared with the control, which indicated that the expression of the gene was closely related to the cold tolerance of the seedlings (Fig. 4).

The wilting rate of the seedlings was observed under different salt stress. The results showed that the wilting rate of the seedlings increased in $0-6 \mathrm{~h}$. There was a positive correlation between wilting rate and salt concentration, the wilting rate of the seedlings treated with different salt concentration reached the maximum at $6 \mathrm{~h}$. After $6 \mathrm{~h}$, the wilting rate declined and returned to normal within $48 \mathrm{~h}$. This result indicated that the salt tolerance of the L. apetalum seedlings was very strong (Table 3 Suppl.). Under $150 \mathrm{mM} \mathrm{NaCl}$ salt stress, the expression of the $L a A b c 1-3$ gene decreased and tended to be relatively stable during salt stress, it was 0.5 -fold of that of the control group, down-regulation of expression was found after seedling recovery. Under $300 \mathrm{mM} \mathrm{NaCl}$ salt stress, its expression decreased and stabilized, but there was no significant difference compared with the control group (Fig. 5A). The trend of the LaAbc1-21 gene expression under salt stress was consistent with that of wilting rate of single-headed $L$. apetalum seedlings. Under $150 \mathrm{mM} \mathrm{NaCl}$, the gene expression reached its maximum at $12 \mathrm{~h}$, and it was 217 -fold higher than of the control group. With the prolongation of treatment time, the expression of the gene decreased, but still maintained high. There was no significant difference in the expression of the gene between the seedling recovery groups and the control group. The maximum expression of the gene was achieved at $24 \mathrm{~h}$ after $300 \mathrm{mM} \mathrm{NaCl}$ treatment, it was 247-fold of the control group, and with the increase of treatment time the gene still maintained a high expression. It was speculated that the gene played a role in salt stress tolerance of $L$. apetalum seedlings (Fig. 5B).

Different concentrations of PEG 6000 were used to treat the $L$. apetalum seedlings, and the wilting rate of the $L$. apetalum seedlings increased from 0 to $24 \mathrm{~h}$, and it was concentration of PEG 6000 dependent. The wilting rate of seedlings treated with different concentrations of 


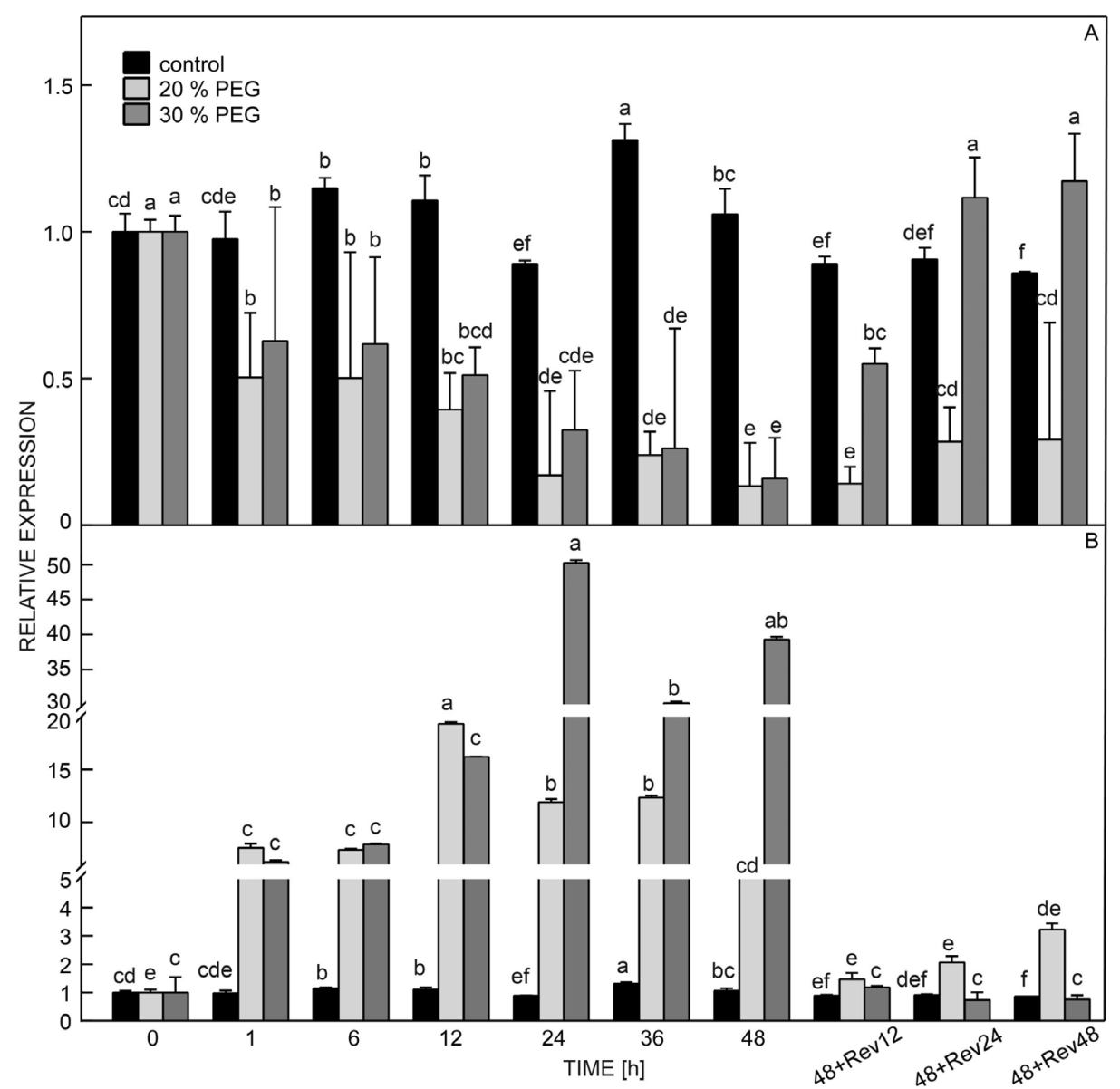

Fig. 6. Lepidium apetalum activity of $b c l$ complex $(A B C 1)$ relative expression in seedlings treated with different concentrations of polyethylene glycol 6000 for 0 to $48 \mathrm{~h}$ and during recovery for 12 to $48 \mathrm{~h}$ (Rev). $A-L a A b c 1-3, B-L a A b c 1-21$. Means $\pm \mathrm{SEs}, n=3$ samples from 40 - 50 plants; different letters indicate significant differences among treatments based on the least significant difference at $P \leq 0.05$.

PEG 6000 reached the maximum at $24 \mathrm{~h}$, and decreased after $24 \mathrm{~h}$, the seedlings returned to normal state after $48 \mathrm{~h}$ only for 10 and $15 \%$ PEG 6000 treatment. The wilting rate of seedlings exposed to other concentrations was still high. Only a few of the seedlings treated with $30 \%$ PEG 6000 failed to recover after PEG 6000 removal, which indicated that the $L$. apetalum seedlings had strong drought tolerance (Table 4 Suppl.). Under the stress conditions caused by 20 and $30 \%$ PEG 6000, the expression of the LaAbcl-3 gene was down-regulated, the trend was more obvious under $30 \%$ PEG 6000, and the expression increased after seedling recovery was resumed. There was no significant difference between the control group and the $30 \%$ PEG 6000 treatment group (Fig. 6A). Under 20 and $30 \%$ PEG 6000, the expression of the LaAbc1-21 gene increased rapidly together with the wilting rate of the seedlings. Under $20 \%$ PEG 6000 treatment, the the LaAbc1-21 gene expression reached the maximum at $12 \mathrm{~h}$, which was 20-times that of the control group, and then the expression level slightly decreased, but the expression was still maintained at a higher level. Under $30 \%$ PEG 6000 treatment, the gene expression reached the maximum at $24 \mathrm{~h}$ which is 50 -times higher than of the control group.
After the recovery, the expression of the gene under the two treatments was not significantly different from that of the control group (Fig. $6 B$ ). It is speculated that the expression of this gene is related to osmotic stress tolerance of the L. apetalum. The low temperature $\left(0{ }^{\circ} \mathrm{C}\right)$, salt (300 mM NaCl), and drought (30\% PEG 6000) treatments showed that $L a A b c 1-3$ had no significant difference in low temperature and salt stress, and it was down-regulated under osmotic stress. It indicated that the expression of the LaAbc1-3 gene had a negative regulation effect on drought tolerance of $L$. apetalum seedlings. LaAbcl-21 was upregulated under low temperature, salt, and osmotic stress, and its response to salt and osmotic stress was significant. It was speculated that the gene was related to abiotic stress tolerance of L. apetalum.

\section{Discussion}

Based on transcriptome sequencing data, researchers have screened members of $A B C 1$ gene family in many plants. For example, there are 15 members in Oryza sativa $L$ (Gao et al. 2011a,b), 19 members in Zea mays L. (Gao 
et al. 2010), and 17 members in Arabidopsis thaliana (Yang et al. 2012a,b,c). Our research team screened $21 A B C 1$ gene members before and after the cessation of low temperature germination. Some $A B C 1$ genes are less variable in expression when $L$. apetalum is at a low-temperature during germination and may change as $L$. apetalum germinates and grows. Therefore, there are not only 21 genes members of $A B C 1$ family in L. apetalum, but the exact number needs to be further studied.

Quantitative analysis of L. apetalum seed fluorescence before and after releasing germination stagnation at low temperature showed that there were 4 up-regulated genes, 6 down-regulated genes, and 11 non-significantly differential genes. Comprehensive analysis found that the expressions of LaAbc1-7, LaAbc1-12, LaAbc1-13, and $L a A b c 1-19$ genes were not significantly different in the low temperature germination of L. apetalum seedlings and the low temperature growth of L. chinensis seedlings. It was speculated that these genes may be insensitive to temperature or constitutively expressed genes. The LaAbc1-1, LaAbc1-4, LaAbc1-11, LaAbc1-14 and $L a A b c 1-17$ genes were not significantly different in low temperature germination of $L$. apetalum seeds, but down-regulated in low temperature growth of L. apetalum seedlings. It is speculated that these genes may reduce the hydrolysis of amino acids by reducing the decomposition of certain proteins during low temperature growth of seedlings, thus reducing ammonia. The expressions of LaAbc1 genes might alleviate plants damage which made $L$. apetalum tolerant to low temperature stress. The expression of the LaAbc1-18 gene was not significantly different during low-temperature germination, but upregulated during low-temperature growth of $L$. apetalum seedlings. It is speculated that this gene was related to cold tolerance of L. apetalum seedlings (Li et al. 2017).

The LaAbc1-10 gene was down-regulated during low temperature germination and seedling growth of $L$. apetalum. It was speculated that the $L a A b c 1-10$ gene might play a negative regulatory role in low temperature stress of L. apetalum. The LaAbc1-9 gene was downregulated during low-temperature germination of L. apetalum seeds, but there was no significant difference in the expression of the $L a A b c 1-9$ gene at the $0{ }^{\circ} \mathrm{C}$ treatment of L. apetalum seedlings. It is speculated that this gene was related to the release of low-temperature germination stagnation of L. apetalum seeds. The LaAbc1-2, LaAbc1-5, and $L a A b c 1-6$ genes were slightly down-regulated during low-temperature germination but up-regulated during lowtemperature growth of the L. apetalum seedlings, which may be related to low-temperature stress tolerance of the L. apetalum seeds and cold tolerance of the seedlings similarly as suggested Yuan et al. (2018).

The LaAbcl-16 and LaAbcl-20 genes were upregulated during low-temperature germination of L. apetalum seeds, but there was no significant difference in the expression of LaAbc1-16 and LaAbc1-20 genes during low-temperature growth of $L$. apetalum seedlings. The LaAbc1-8 and LaAbc1-21 genes were up-regulated during low-temperature germination and seedling growth of $L$. apetalum. It was speculated that these genes might be closely related to low-temperature germination of L. apetalum seeds and cold tolerance of seedlings (Wang et al. 2004).

The expression of the LaAbcl-3 gene increased in order to enhance the adaptability of seedlings to low temperature. After removing low temperature stress, the expression of LaAbcl-3 decreased. It may be that the L. apetalum seedlings synthesized enough proteins to tolerate low temperature stress and resumed control culture. After removing low temperature stress, the expression of the LaAbc1-3 gene was up-regulated. It is speculated that in the L. apetalum seedlings under the influence of low temperature stress, physiological regulation did not return to normal. The expression of the $L a A b c 1-21$ gene decreased first and then increased under low temperature stress. The decrease of $L a A b c 1-21$ gene expression may be due to the damage of physiological characteristics of the seedlings, which inhibited the expression of the LaAbcl-21 gene. After a period of time, the $L a A b c 1-21$ gene enhances plant tolerance to low temperature by increasing expression ( $\mathrm{Li}$ et al. 2017, Yuan et al. 2018, Zhao et al. 2018).

The LaAbc1-3 gene was insensitive to salt stress. The expression of $L a A b c 1-21$ gene was significantly up-regulated under salt stress, and the higher the salt concentration, the more obvious the up-regulated trend was. The expression trend of the LaAbc1-21 gene was consistent with the wilting rate of $L$. apetalum seedlings under salt treatment, which indicated that the expression of the LaAbc1-21 gene might be related to salt tolerance of L. apetalum seedlings (Yang et al. 2012a). Members of this family are involved in a wide range of abiotic stress responses and may be related to plant resistance to stress. At the same time, we noticed that the expression patterns of LaAbc1-3 and LaAbc1-21 genes were different under salt stress, indicating that homologous genes can perform different functions in different species.

Under osmotic stress, the expression of the LaAbcl-3 gene was down-regulated, the higher the concentration of PEG 6000, the more obvious was the down regulation trend of the gene. This result indicated that osmotic stress inhibited the expression of the LaAbcl-3 gene, and the expression was negatively correlated with the PEG 6000 concentration. The expression of the $L a A b c 1-21$ gene was significantly up-regulated under osmotic stress, and the expression was positively correlated with the concentration of PEG. The trend of the LaAbc1-21 gene expression was consistent with the wilting rate of $L$. apetalum seedlings, suggesting that the gene expression might be related to drought tolerance of $L$. apetalum seedlings (Wang et al. 2011). Studies have shown that $A B C 1$ genes in Lilium regale do not respond to drought stress (Yuan et al. 2014), but four $O s A B C 1$ genes in rice are inhibited by drought stress (Gao et al. 2011a,b). In this study, the expression patterns of LaAbc1-3 and LaAbc1-21 genes are different, indicating that the expression of $A B C 1$ gene can be induced by a variety of abiotic stresses and may perform different biological functions in different species.

The changes of LaAbc1-3 under the abiotic stresses of $L$. apetalum were not very obvious, but $L a A b c 1-3$ had certain tissue specificity during the growth and 
development of $L$. apetalum. The LaAbcl-3 was upregulated in the old stem of $L$. apetalum., but the expression in the other organs did not change significantly (Fig. 7 Suppl.). The ABC1 gene family has obvious tissue and organ expression specificity. Among them, the $14 \mathrm{ABC1}$ gene family members of rice have the highest expression in leaves (Gao et al. 2011a,b). In wheat, the expression of this gene is mainly concentrated in green tissues such as leaves and stem tips (Gao et al. 2010). It is speculated that this gene may be related to plant growth and development. LaAbc1-21 has obvious changes in L. apetalum under abiotic stresses. It is speculated that this gene plays an important role in L. apetalum abiotic stress tolerance, especially to salt stress and osmotic stress. Although both genes have the same aarF domain, they show different roles in biological functions, indicating that this domain may not be a key factor for the $A B C 1$ gene family to cope with abiotic stresses.

These results suggest that LaAbcl expression, especially the LaAbc1-21 gene, might play an important role in the tolerance of $L$. apetalum seedlings to a low temperature, salinity, and osmotic stress although additional experimental evidence is needed to determine whether $L a A b c 1-21$ increases the ability of plants to retain water. The above analysis indicates that $L$. apetalum $\mathrm{ABC} 1$ family not only plays a role in stress response, but may also be related to specific physiological processes in L. apetalum.

\section{References}

Bousquet, I., Dujardin, G., Slonimski, P.P.: $A B C 1$, a novel yeast nuclear gene has a dual function in mitochondria: it suppresses a cytochrome $b$ mRNA translation defect and is essential for the electron transfer in the bc 1 complex. - EMBO J. 10: 20232031, 1991.

Do, T.Q., Hsu, A.Y., Jonassen, T., Lee, P.T., Clarke, C.F.: A defect in coenzyme $\mathrm{Q}$ biosynthesis is responsible for the respiratory deficiency in Saccharomyces cerevisiae abcl mutants. J. biol. Chem. 276: 18161-18168, 2001.

Ernster, L., Forsmark, A.P.: Ubiquinol: an endogenous antioxidant in aerobic organisms. - Clin. Invest. 71: S60-S65, 1993.

Gao, Q., Yang, Z., Zhou, Y., Yin, Z., Qiu, J., Liang, G.: Characterization of an $\mathrm{ABC} 1$ kinase family gene os $A B C 1-2$ conferring enhanced tolerance to dark-induced stress in rice. Gene 498: 155-163, 2012.

Gao, Q.S., Yang, Z.F., Zhou Y., Zhang, D., Yan, C.H., Liang, G.H., Xu, C.W.: Cloning of a maize ABC1 gene ZmABC1-10 and its response to abiotic stresses such as cadmium. - J. Crops 12: 2073-2083, 2010.

Gao, Q.S., Zhang, D., Xu, L., Xu, C.W.: Systematic identification of rice $A B C 1$ gene family and its response to abiotic stress. Rice Sci. 3: 14-24. 2011 a.

Gao, Q.S., Zhang, D., Xu, L., Xu, C.W.: Identification of rice $A B C 1$ gene family and expression analysis under abiotic stress. - Chin. J. Rice Sci. 1: 1-10. 2011 b.

Hsieh, E.J., Dinoso, J.B., Clarke, C.F.: A trnatrp gene mediates the suppression of cbs2-223 previously attributed to abc1/ coq8. - Biochem. biophys. Res. Commun. 317: 648-653, 2004.

Jasinski, M., Sudre, D., Schansker, G., Schellenberg, M., Constant, S., Martinoia, E.: AtOSA1, a member of the ABC1- like family, as a new factor in cadmium and oxidative stress response. - Plant Physiol. 147: 719-731, 2008.

Leonard, C.J., Aravind, L., Koonin, E.V.: Novel families of putative protein kinases in bacteria and archaea: evolution of the "eukaryotic" protein kinase superfamily. - Genome Res. 8: 1038-1047, 1998.

Li, L.L., Zeng, W.J., Li, Y.H., Ge, F.W., Lu, H., Lei, W., Du, Y., Xie, H.T., Zhao, H.P., Zhao, H.X.: Analysis of GRAS transcription factor family and cloning of LaSCL18 gene in Lepidopsis uniquely and its cold-related study. - Mol. Plant Breed. 15: 3428-3437, 2017.

Li, P.P., Zeng, W.J., Zhou, Q., Zhao, H.X., Li, Y.H., Ge, F.W., Zhu, C.Q., Zhao, J.J., Lu, H., Zhao, H.P.: Screening and expression analysis of temperature response proteins to remove the stagnation of low temperature germination of Lepidopsis uniflora seeds. - Mol. Plant Breed. 14: 722-729, 2016.

Macinga., D.R., Cook, G.M., Poole, R.K., Rather, P.N.: Identification and characterization of aarf, a locus required for production of ubiquinone in Providencia stuartii and Escherichia coli and for expression of 2'-N-acetyltransferase in P. stuartii. - J. Bacteriol. 180: 128-135, 1998.

Mao, Z.M., Zhang, X.M.: Flora outline of early spring short-lived plants in northern Xinjiang. - Arid Zone Res. 11: 1-26, 1994.

Meng, J., Li, Q., Li, G.: Study on the physiological characteristics of seed germination of two species of Lepidopsis. Biotechnology 18: 32-35, 2008.

Mollet, J., Delahodde, A., Serre, V., Chretien, D., Schlemmer, D., Lombes, A., Boddaert, N., Desguerre, I., De Lonlay, P., De Baulny, H.O., Munnich, A., Rötig, A.: $C A B C 1$ gene mutations cause ubiquinone deficiency with cerebellar ataxia and seizures. - Amer. J. Human Genet. 82: 623-630, 2008.

Smita, S., Katiyar, A., Lenka, S.K., Dalal, M., Kumar, A., Mahtha, S.K.: Gene network modules associated with abiotic stress response in tolerant rice genotypes identified by transcriptome meta-analysis. - Funct. integr. Genomics 20: 29-49, 2020.

Tamura, K., Peterson, D., Peterson, N., Stecher, G., Nei, M., Kumar, S.: MEGA5: molecular evolutionary genetics analysis using maximum likelihood, evolutionary distance, and maximum parsimony methods. - Mol. Biol. Evol. 28: 27312739, 2011.

Tauche, A., Krause, B.U., Rodel, G.: Ubiquinone biosynthesis in Saccharomyces cerevisiae: the molecular organization of $o$-methylase coq $3 p$ depends on abclp/coq 8 p. - FEMS Yeast Res. 8: 1263-1275, 2013.

Thompson, J.D., Plewniak, F., Poch, O.: A comprehensive comparison of multiple sequence alignment programs. - Nucl. Acids Res. 27: 2682-2690, 1999.

Trumpower, B.L.: New concepts on the role of ubiquinone in the mitochondrial respiratory chain. - J. Bioenerg. 13: 1-24, 1981.

Trumpower, B.L.: Cytochrome $b c 1$ complexes of microorganisms. - Microbiol. Rev. 54: 101-129, 1990.

Villalba, J.M., Navas, P.: Plasma membrane redox system in the control of stress-induced apoptosis. - Antioxid. Redox Signaling 2: 213-230, 2000.

Wang, C.X., Jing, R.L., Mao, X.G., Chang, X.P., Li, A.: TaABC1, a member of the activity of bc 1 complex protein kinase family from common wheat, confers enhanced tolerance to abiotic stresses in Arabidopsis. - J. exp. Bot. 62: 1299-1311, 2011.

Wang, C.X., Jing, R.L., Mao, X.G., Pang, X.B., Liu, H.M., Chang, X.P.: Cloning and expression analysis of wheat TaABC1L. - J. Crops. 33: 878-884, 2004.

Yang, N., Zhao, H.P., Ge, F.W., Li, Y.H., Zeng, W.J., Zhao, H.X.: Physiological response of germination of two species of Lonicerae to low temperature stress. - Arid Zone Res. 32: 760-765, 2015.

Yang, Q.L., Lu, H., Zhou, Q., Xie, H.T., Li, J.Y., Du, Y., Chen, 
Z.Y., Han, S.C., Zhao, H.P., Zhao, H.X.: Analysis of LEA protein family members in Lepidium apetalum seeds and the expression of LaLEA1 in seedlings in response to abiotic stresses. - Biol. Plant. 64: 211-219, 2020.

Yang, S.G., Li, T., Liu, M., Gao, S.J., Yang, S.Y., Li, L.: Phylogenetic, structure and expression analysis of ABC1ps gene family in rice. - Biol. Plant. 56: 667-674, 2012a.

Yang, S.G., Zeng, X.Q., Li, T., Liu, M., Zhang, S.H., Gao, S.J., Wang, Y.Q., Peng, C.L., Li, L., Yang, C.W.: Atacdo1, an ABCl-like kinase gene, is involved in chlorophyll degradation and the response to photooxidative stress in Arabidopsis. - J. exp. Bot. 63: 3959-3973, 2012b.

Yang, S., Zhang, Q., Li, T., Du, J., Yang, S., Yang, C.: AtSIA1, an ABC1-like kinase, regulates salt response in Arabidopsis. - Biológia 67: 1107-1111, 2012c.

Yuan, L.L., Wang, Y.R., Zeng, W.J., Xie, H.T., Du, Y., Lu, H., Zhao, H.X.: Response of $b H L H$ transcription factor gene family and laICE1 expression in seedlings of uninhabited vegetable to cold stress. - Acta bot. boreali-occident. sin. $\mathbf{3 8}$ : 26-34, 2018.

Yuan, Y.Y., Liang, Y., Yuan, S.X., Xu, L.F., Li, Y.N., Younes, P.H., Liu, C., Ming, J.: Cloning of the lily $A B C 1$ protein kinase gene and its expression patterns under abiotic stresses in Lilium regale. - Acta hort. sin. 41: 2246-2454, 2014.

Young, B.M., Hua, C.L., Jungeun, L., Hyun, P., Andosung, L., Taek, K.W., Hyoungseok, L.: Identification of rice genes associated with enhanced cold tolerance by comparative transcriptome analysis with two transgenic rice plants overexpressing dacbf4 or dacbf7, isolated from antarctic flowering plant Deschampsia antarctica. - Front. Plant Sci. 9: 601-610, 2018.

Zhao, H.X., Li, Q., Jin, Z.: Tolerance to low temperature during seed germination of short-lived plants. - Plant Diversity Resour. 32: 448-454, 2010.

Zhao, H.X., Zhao, Q.Q., Lu, H., Zhu, Y.L., Yu, L.J., Qian, H.B., Du, Y., Xie, H.T., Zhao, W.J., Zeng, W.J.: Response of DREB transcription factor family and $L a D R E B$ expression to cold stress in seedlings of Lepidopsis uniquely. - Mol. Plant Breed. 16: 42-48, 2018.

Zhou, Q., Zhao, H.X., Li, P.P., Zeng, W.J., Li, Y.H., Ge, F.W., Zhao, J.J., Zhao, H.P.: High-throughput sequencing and analysis of the transcriptome of the seeds of Lepidopsis uniflora. - China Biotechnol. 36: 38-46, 2016. 